\title{
Poor 'real-life' negative predictive value of cross-sectional imaging in obstructive jaundice
}

\author{
Brian A Brunson $M D^{1}$, Robert Hawes $\mathrm{MD}^{2}$, Brenda Hoffman $\mathrm{MD}^{3}$, Stacie Vela $\mathrm{MD}^{4}$, Joseph Romagnuolo $\mathrm{MD}^{5}$
}

BA Brunson, R Hawes, B Hoffman, S Vela, J Romagnuolo. Poor 'real-life' negative predictive value of cross-sectional imaging in obstructive jaundice. Can J Gastroenterol Hepatol 2014;28(7):385-390.

BACKGROUND: Cross-sectional imaging remains the first-line test for obstructive jaundice despite high miss rates for pancreatobiliary tumours. Improvements in resolution and slice thickness of spiral computed tomography/magnetic resonance imaging/magnetic resonance cholangiopancreatography promised to increase accuracy.

OBJECTIVE: To assess whether the post-test probability of neoplasm is truly altered by the presence or absence of a mass on computed tomography/magnetic resonance imaging in obstructive jaundice.

METHODS: The institutional endoscopic ultrasound (EUS) database was retrospectively reviewed to stratify patients presenting to EUS over a two-year period for obstructive jaundice (suspicious for malignancy) according to their pre-EUS imaging results. The primary analysis involved the calculation of the positive predictive value and negative predictive value (NPV) of imaging with 95\% binomial CIs. Test performance of EUS/fine-needle aspiration (FNA) was also calculated. Final diagnosis was determined by positive cytology/histology; negative EUS was supplemented by clinical follow-up.

RESULTS: The positive predictive value $(n=51)$ and NPV $(n=53)$ of pre-EUS imaging was $98 \%$ (95\% CI 90\% to 100\%) and 9\% (95\% CI $3 \%$ to $21 \%$ ), respectively (accuracy $53 \%$ ), with post-test suspicion of malignancy similar between imaging-positive and -negative groups. EUS demonstrated a mass in $96 \%$ of imaging-positive cases versus $85 \%$ in imaging-negative cases (exact $\mathrm{P}=0.09$ ). Malignant or suspicious FNA cytology was obtained with EUS in $92 \%$ of the imaging-positive group, and $62 \%$ of the imaging-negative group ( $75 \%$ of subgroup with FNA) $(\mathrm{P}<0.001)$.

CONCLUSION: Lack of a definite mass on pre-EUS imaging had low NPV, and was clearly not sufficiently accurate or reassuring in this clinical setting. In suspicious obstructive jaundice, EUS with FNA has a high diagnostic yield regardless of the findings of pre-EUS cross-sectional imaging and, as such, EUS may be a more reasonable first-line test in this high-suspicion setting.

Key Words: CT; Diagnostic test accuracy; EUS; MRI; Obstructive jaundice; Pancreatic cancer; Predictive value
La mauvaise valeur prédictive négative de l'imagerie transversale en milieu réel en cas de jaunisse obstructive

HISTORIQUE : L'imagerie transversale demeure le test de première ligne de la jaunisse obstructive, malgré le taux élevé de tumeurs pancréatobiliaires non décelées. L'amélioration de la résolution et de l'épaisseur des coupes de la tomodensitométrie spiralée, de l'imagerie par résonance magnétique ou de la cholangiopancréatographie par résonance magnétique promet d'accroître la précision des résultats.

OBJECTIF : Évaluer si la présence ou l'absence de masse à la tomodensitométrie ou l'imagerie par résonance magnétique modifie vraiment la probabilité de néoplasme après le test en cas de jaunisse obstructive.

MÉTHODOLOGIE : Les chercheurs ont procédé à l'examen rétrospectif de la base de données des échographies endoscopiques (ÉGE) de l'établissement pour stratifier les patients qui avaient subi une ÉGE sur une période de deux ans en raison d'une jaunisse obstructive (qui pouvait être maligne) compte tenu des résultats de l'imagerie avant l'ÉGE. Pendant l'analyse primaire, ils ont calculé la valeur prédictive positive et la valeur prédictive négative (VPN) de l'imagerie au moyen d'indices de confiance binomiaux de $95 \%$. Ils ont également calculé le rendement de l'ÉGE et de l'aspiration à l'aiguille fine (AAF). Ils ont déterminé le diagnostic définitif par cytologie ou histologie. L'ÉGE négative était complétée par le suivi clinique.

RÉSULTATS : La valeur prédictive positive $(\mathrm{n}=51)$ et la VPN $(\mathrm{n}=53)$ de l'imagerie avant l'ÉGE s'élevait à $98 \%$ (IC $90 \%$ à $100 \%$ ) et à $9 \%$ (IC $3 \%$ à $21 \%$ ), respectivement (exactitude de $53 \%$ ), et la suspicion de cancer après le test était similaire dans les groupes dont l'imagerie était positive ou négative. L'ÉGE a révélé une masse chez $96 \%$ des cas positifs à l'imagerie, par rapport à $85 \%$ des cas dont l'imagerie était négative (exact $\mathrm{P}=0,09)$. Les résultats de cancer ou de suspicion de cancer par l'aspiration cytologique à l'aiguille fine ont été obtenus à l'aide de l'ÉGE chez $92 \%$ des membres du groupe positif à l'imagerie et chez $62 \%$ de ceux du groupe négatif à l'imagerie ( $75 \%$ du sous-groupe ayant subi une AAF) $(\mathrm{P}<0,001)$. CONCLUSION : L'absence de masse définie à l'imagerie avant l'ÉGE s'associait à une faible VPN. De toute évidence, elle n'était pas assez précise ou rassurante dans ce contexte clinique. En cas de jaunisse obstructive faisant croire à un cancer, l'ÉGE accompagnée de l'AAF s'associe à un rendement diagnostique élevé, quels que soient les résultats de l'imagerie transversale avant l'ÉGE. Ainsi, il est peut-être plus raisonnable d'utiliser l'ÉGE en première ligne dans ce contexte de soupçon élevé. ancreatic cancer is the fourth leading cause of cancer-related death
in the United States, with an estimated incidence of 37,700 cases
2008 (1). Smaller pancreatic tumours can be asymptomatic and others,
despite modest size, are simply invisible to conventional imaging,
resulting in delays in diagnosis and a later stage at the time of treat-
ment planning ( 2 ).
Cross-sectional imaging using computed tomography (CT) or mag-
netic resonance (MR) imaging is generally still recommended first
when there is high suspicion (1,3). However, because pancreatic
tumours are often isodense/isointense on noncontrast CT/MR imaging,
one must often rely on hypoperfusion of contrast to highlight the tumour, but hypoperfusion is not a universal feature of these tumours. The reported accuracy ranges from $67 \%$ to $84 \%(2,4-6)$ for contrast $\mathrm{CT} / \mathrm{MR}$ in pancreatobiliary tumours. For CT, the sensitivity ranges from as low as $53 \%$ to as high as $92 \%$, and the specificity is $40 \%$ to $70 \%$. For MR imaging, Muller et al (4) showed good overall accuracy, but for tumours $<3 \mathrm{~cm}$ in size $(\mathrm{n}=15)$, accuracy dropped to $67 \%$ (and to $53 \%$ for CT), while EUS sensitivity remained $>90 \%$. A positive CT/MR image when jaundice is absent is equally unreliable (7). Despite advances in technology (resolution, sequences and slice thickness) (8), the negative predictive value (NPV) of spiral CT was still $<30 \%$ in a more recent study by Agarwal et al (2) and the 'real-life'

${ }^{1}$ Gastroenterology Associates, Birmingham, Alabama; ${ }^{2}$ Florida Hospital, Orlando, Florida; ${ }^{3}$ Department of Medicine, Medical University of South

Carolina, Charleston, South Carolina; ${ }^{4}$ Baylor Medical College, Houston, Texas; ${ }^{5}$ Palmetto Health and Columbia Gastroenterology Associates,

Columbia, South Carolina, USA.

Correspondence: Dr Joseph Romagnuolo, Palmetto Health and Columbia Gastroenterology Associates, 2739 Laurel Street, Suite 1A, Columbia,

South Carolina 29204, USA. Telephone 803-799-4800, fax 803-252-0052, e-mail romagnuoloj@gmail.com

Received for publication April 3, 2014. Accepted April 19, 2014 
TABLE 1

Demographics, clinical data, findings and final diagnosis stratified according to pre-endoscopic ultrasound (EUS) imaging positive or negative for a mass

\begin{tabular}{|c|c|c|c|}
\hline & \multicolumn{2}{|c|}{ Mass on pre-EUS imaging } & \multirow[b]{2}{*}{$\mathbf{P}$} \\
\hline & Yes $(n=51)$ & No $(n=53)$ & \\
\hline Age, years, mean (range) & $65(38-80)$ & $67(43-86)$ & 0.32 \\
\hline Male sex & $28(55)$ & $30(57)$ & 0.86 \\
\hline \multicolumn{4}{|l|}{ Presentation/symptoms } \\
\hline Obstructive jaundice & $51(100)$ & $53(100)$ & 1.0 \\
\hline Abdominal pain & $14(27)$ & $13(25)$ & 0.73 \\
\hline Painless & $37(73)$ & $40(75)$ & 0.73 \\
\hline Stricture at ERCP & $28(55)$ & $36(68)$ & 0.17 \\
\hline Chronic pancreatitis & $3(6)$ & $6(11)$ & 0.49 \\
\hline \multicolumn{4}{|l|}{ Pre-EUS imaging } \\
\hline Magnetic resonance & $2(4)$ & $3(6)$ & 1.0 \\
\hline $\mathrm{MRCP}$ & $10(20)$ & $20(38)$ & 0.04 \\
\hline Computed tomography & $44(86)$ & $41(77)$ & 0.24 \\
\hline Imaging in-house & $7(14)$ & $2(4)$ & 0.09 \\
\hline \multicolumn{4}{|l|}{ Pre-EUS imaging results } \\
\hline Mass & $51(100)$ & $0(0)$ & $\mathrm{N} / \mathrm{A}$ \\
\hline Possible mass/'fullness' & $0(0)$ & $9(17)$ & $\mathrm{N} / \mathrm{A}$ \\
\hline Chronic pancreatitis & $1(2)$ & $0(0)$ & 0.49 \\
\hline Duct dilation & $39(76)$ & $38(72)$ & 0.007 \\
\hline Cysts & $2(4)$ & $1(2)$ & 0.61 \\
\hline \multicolumn{4}{|l|}{ EUS findings } \\
\hline Mass & $49(96)$ & $45(85)$ & 0.09 \\
\hline Short-axis, mm, mean (range) & $24.7(8.0-59)$ & $20.8(6.3-37)$ & 0.07 \\
\hline Bile duct wall thickening only & $0(0)$ & $2(4)$ & 0.50 \\
\hline Chronic pancreatitis ( $\geq 5$ criteria) & $6(12)$ & $8(15)$ & 0.78 \\
\hline \multicolumn{4}{|l|}{ Staging (if available) } \\
\hline T1/T2 lesions & $5(9.8)$ & $4(7.5)$ & 0.73 \\
\hline T3 without vascular invasion & $22(43)$ & $25(47)$ & 0.70 \\
\hline T4 or T3 with vascular invasion & $18(35)$ & $12(23)$ & 0.20 \\
\hline N1 & $22(43)$ & $15(28)$ & 0.15 \\
\hline \multicolumn{4}{|l|}{ Fine-needle aspiration results } \\
\hline Not performed & $2(4)$ & $9(17)$ & 0.052 \\
\hline Malignant & $37(73)$ & $23(43)$ & 0.003 \\
\hline Suspicious & $10(20)$ & $10(19)$ & 0.92 \\
\hline Atypical & $1(2)$ & $5(9)$ & 0.21 \\
\hline Negative/benign & $1(2)$ & $6(11)$ & 0.11 \\
\hline Passes, n, mean (range) & $5.3(2-10)$ & $5.0(2-10)$ & 0.78 \\
\hline \multicolumn{4}{|l|}{ Final diagnosis } \\
\hline Malignant & $50(98)$ & $48(91)$ & 0.21 \\
\hline Benign & $1(2)$ & $5(9)$ & 0.21 \\
\hline Chronic pancreatitis & $1(2)$ & $2(4)$ & 1.0 \\
\hline Autoimmune pancreatitis & 0 & $1(2)$ & 1.0 \\
\hline False-negative EUS & $2(4)$ & $8(15)$ & 0.09 \\
\hline
\end{tabular}

Data presented as $n$ (\%) unless otherwise indicated. ERCP Endoscopic retrograde cholangiopancreatography; MRCP Magnetic resonance cholangiopancreatography. N/A Not applicable

effectiveness of community interpretation may be even lower. This does not appear to be a resolution problem, but rather an inherent shortcoming of the imaging modalities themselves. However, larger studies of miss rates of CT/MR imaging in high-suspicion cohorts have not been published.

Endoscopic ultrasound (EUS) is well-established for the detection of pancreatic tumours, especially small ones (2,6,9-12). Tumours more consistently have a different echogenicity than surrounding normal pancreas on EUS and fine-needle aspiration (FNA) helps clarify/confirm findings. The reported sensitivity, specificity, accuracy and predictive values of EUS-FNA are $>90 \%$ in multiple studies
(2,13-17). Even in a moderately high-prevalence setting, FNA has high NPV (18). The continued widespread use of CT/MR first in high-suspicion cases can either give false reassurance to internists and delay a diagnosis, or false reassurance regarding small size and resectability to surgeons (ie, one cannot stage what one cannot see).

Nearly all high-suspicion patients are ultimately sent to EUS from our referral base, either to clarify (negative imaging) or confirm/stage (positive imaging) the diagnosis, leading to two natural substrata of patients. Our aim was to evaluate the real-life effectiveness (predictive values) of cross-sectional imaging in obstructive jaundice by evaluating the rate of cancer diagnosis, stratified according to whether a tumour was apparent.

\section{METHODS}

Study design

After institutional review board approval, consecutive patients undergoing EUS from September 2007 to July 2009 at the authors' tertiary institution (Medical University of South Carolina, Charleston, South Carolina, USA), for the indication of obstructive jaundice suspicious of pancreatobiliary malignancy (eg, lack of significant pain, unexplained weight loss and/or biliary stricture) were retrospectively reviewed after identification in the endoscopy database. Patients underwent EUS-FNA performed by one of four experienced endosonographers. Exclusion criteria included cystic lesions and other known medical cause for jaundice.

Demographics (age, sex, race, medical record number), symptoms (jaundice, abdominal pain, weight loss or none), CT/MR imaging results (mass, 'fullness'/'enlargement', biliary/pancreatic duct dilation, metastatic disease, calcifications), patient history (recurrent/chronic pancreatitis, alcohol abuse, jaundice), pre-EUS endoscopic retrograde cholangiopancreatography (ERCP) findings/intervention (eg, stenting), EUS scope (eg, radial versus linear) and findings (chronic pancreatitis, probable/possible mass, tumour size/stage) and FNA results were collected. When possible, additional data from the electronic medical record (eg, scanned clinical/radiological data from referring physicians) were reviewed.

\section{EUS}

Linear EUS (without radial) was performed in all but one case (Olympus America, USA). FNA of suspicious masses was performed whenever feasible and indicated. On-site cytopathology was used. For EUS (without FNA) test performance, all (probable/possible) focal 'masses' identified on EUS were considered to be 'positive'.

\section{Final diagnosis and follow-up}

A diagnosis of cancer was defined as positive/suspicious cytology or surgical pathology; 'atypical' cytology was considered to be negative for analysis. In patients with a negative EUS/FNA, one-year clinical followup was sought to rule out disease progression or development of metastatic disease: a composite of subsequent medical records (clinic visits, imaging, procedures [including subsequent EUS or ERCP], operative data and pathology data) and telephone contact (when medical record follow-up not available or equivocal), using an institutional review board-approved phone script, and Authorization to Disclose Protected Health Information form, as needed to obtain outside records.

\section{Sample size and statistical analysis}

A precision-based method was used and showed that 50 positive- and negative-scan patients would correspond to a $\pm 10 \% 95 \%$ binomial CI for positive predictive value (PPV), assuming a predictive value near $90 \%$. The primary analysis included calculation of the PPV and NPV of pre-EUS imaging with $95 \% \mathrm{CI}$, as well as other test performance characteristics for EUS and FNA. Baseline characteristics of the imaging-positive and -negative groups were compared using $\chi^{2}$ or Fisher's exact tests for proportions, and $t$ tests or Mann-Whitney for normal and non-normal continuous measures, respectively (STATA version 7, StataCorp, USA). 
TABLE 2

Test performance characteristics of computed tomography (CT)/magnetic resonance imaging (MRI), endoscopic ultrasound (EUS) and EUS fine-needle aspiration (FNA) for pancreatobiliary malignancy

\begin{tabular}{|c|c|c|c|}
\hline & $\begin{array}{l}\text { Pre-EUS cross-sectional } \\
\text { imaging }(C T / M R I)(n=104)\end{array}$ & EUS (n=104) & EUS-FNA ( $n=93)$ \\
\hline Sensitivity & $51(41-61) ; n=50 / 98$ & 95 (88-98); n=92/97 & 90 (82-95); n=80/89 \\
\hline Specificity & $83(36-100) ; n=5 / 6$ & $71(29-96) ; n=5 / 7$ & $100(40-100) ; n=4 / 4$ \\
\hline Positive predictive value & $98(90-100) ; n=50 / 51$ & 98 (93-100); n=92/94 & $100(95-100) ; n=80 / 80$ \\
\hline Negative predictive value & $9(3-21) ; n=5 / 53$ & 50 (19-81); n=5/10 & $31(9-61) ; n=4 / 13$ \\
\hline Accuracy & 53 (43-63); $n=55 / 104$ & 93 (87-97); n=97/104 & 90 (82-95); n=84/93 \\
\hline
\end{tabular}

Data presented as \% (95\% Cl)

TABLE 3

Test performance characteristics of endoscopic ultrasound (EUS) and EUS fine-needle aspiration (FNA) stratified according to pre-EUS imaging results

\begin{tabular}{|c|c|c|c|c|}
\hline & \multicolumn{2}{|c|}{ Pre-EUS imaging-positive group $(n=51)$} & \multicolumn{2}{|c|}{ Pre-EUS imaging-negative group $(n=53)$} \\
\hline & EUS & EUS-FNA $(n=49)$ & EUS & EUS-FNA $(n=44)$ \\
\hline Sensitivity & 98 (89-100); n=49/50 & $96(86-100) ; n=47 / 49$ & $91(80-98) ; n=43 / 47$ & 83 (67-93); n=33/40 \\
\hline Specificity & $100(2-100) ; n=1 / 1$ & $0(0-0) ; n=0 / 0$ & 67 (22-96); n=4/6 & $100(40-100) ; n=4 / 4$ \\
\hline Positive predictive value & $100(93-100) ; n=49 / 49$ & 100 (92-100); n=47/47 & 96 (85-99); n=43/45 & $100(89-100) ; n=33 / 33$ \\
\hline Negative predictive value & 50 (1-99); n=1/2 & $0(0-84) ; n=0 / 2$ & 50 (16-84); n=4/8 & 36 (11-69); n=4/11 \\
\hline Accuracy & $98(90-100) ; n=50 / 51$ & $96(86-100) ; n=47 / 49$ & 89 (77-96); n=47/53 & $84(70-93) ; n=37 / 44$ \\
\hline
\end{tabular}

Data presented as \% (95\% Cl)

\section{RESULTS}

Patient characteristics

The database search identified 111 patients over the two-year period. After manual review, six patients were excluded: five with previously known pancreatic cyst/pseudocyst and one due to cirrhosis being the most likely pre-imaging etiology of jaundice, leaving 104 patients in the final analysis (Table 1). The mean age for the study patients was 66 years, with $56 \%$ men. All patients presented with obstructive jaundice, which was primarily painless $(74 \%)$ and $62 \%$ had biliary strictures at ERCP (before EUS), mostly drained using a plastic stent before EUS.

The imaging-positive group consisted of 51 patients with a mass clearly apparent on pre-EUS imaging. The imaging-negative group consisted of the remaining 53 patients without a definite pancreaticobiliary mass on pre-EUS imaging. Within this group, seven patients had no abnormalities on imaging, while the remaining 46 had equivocal imaging, with no mass apparent but other findings noted: 'fullness' or 'prominence' of the head in 17\% (nine of 53); ductal dilation in $72 \%$ (38 of 53); and cysts in one patient. Pre-EUS imaging included CT alone in 66\%, MR imaging in 18\% and both in $15 \%$. MR imaging included MR cholangiopancreatography (MRCP) in 30 of 35 cases $(86 \%)$. Nine patients $(9 \%)$ underwent their pre-EUS imaging at the authors' institution, the rest elsewhere. Although some of the details of community and tertiary (including from Columbia, South Carolina, Atlanta, Georgia, and others) CT/MR protocols were not systematically recorded prospectively in the medical records, the examinations were all performed for the indication 'obstructive jaundice' or a 'suspicious biliary stricture'; therefore, most radiology centres generally would have attempted a pancreatic protocol study, often with thin slices through the pancreas. No patient was noted to have had previous EUS or positron emission tomography. The average mass size (range) in cross-section at EUS was $27.5 \mathrm{~mm}(7 \mathrm{~mm}$ to $50 \mathrm{~mm}) \times$ $22.8 \mathrm{~mm}(6 \mathrm{~mm}$ to $40 \mathrm{~mm})$. The average number of FNA passes performed to acquire an adequate sample was five; $94 \%$ FNAs were positive.

Predictive values of pre-EUS imaging

Of the 51 patients with a mass clearly apparent on pre-EUS imaging, 50 were diagnosed with a malignancy, yielding a PPV of 98\% (95\% CI $90.0 \%$ to $100.0 \%$ ) (Table 2 ). In the 53 cases with no clear mass on pre-EUS imaging, malignancy was only diagnosed slightly less often, with 48 of these patients diagnosed with a malignant neoplasm, yielding a false-negative rate of 91\% (ie, an NPV of only 9.4\% [95\% CI
$3.1 \%$ to $20.7 \%]$ ). That is, the chance of finding malignancy was similar regardless of whether mass was apparent on cross-sectional imaging (98\% versus 91\%) (Figure 1).

To further clarify the possible relevance of equivocal pre-EUS imaging in the group of patients without a clear mass on imaging, a post hoc secondary analysis was performed, separating completely normal imaging from equivocal imaging. Of the seven patients with completely normal imaging, four $(57 \%)$ progressed to have malignant or suspicious cytology; two others (thickening of the common bile duct on imaging with upstream dilation) had benign cytology and benign follow-up; and the remaining patient had a benign clinical course with resolution of jaundice, yielding a malignancy rate of $57 \%$. Of those with 'equivocal imaging' (eg, 'fullness'), 42 of the 46 (91\%) were ultimately diagnosed with malignancy. Therefore, completely normal imaging had a slightly higher NPV than had equivocal imaging (without a definite mass), but the former's NPV remained $<50 \%$.

Although tumour size may play a role in accuracy, the tumours ultimately seen in the pre-EUS imaging-negative/equivocal group were only slightly smaller $(<5 \mathrm{~mm}$ smaller than the positive group, $\mathrm{P}=0.07$ ), with similar range. Assuming pretest probability of cancer of $90 \%$ in obstructive jaundice, the likelihood ratio of positive CT or MR imaging was calculated to be 5.4, but for a negative study was 1.1 (in which a likelihood ratio of 1.0 represents an unhelpful test, not changing pre-test probability). The sensitivity and accuracy of preEUS imaging were $51 \%$ and $53 \%$, respectively.

Setting or quality of pre-EUS imaging may be relevant. It is notable that of the nine imaging studies that were performed at the institution, eight of nine were performed with contrast: three had CT with contrast alone; two had both CT and MRCP with contrast; three had MR imaging/MRCP with contrast; and one had MRCP alone without contrast. Of these nine, seven were positive (and all confirmed positive by EUS [100\% PPV]) and two were negative (both confirmed to be false-negatives by EUS, finding small periampullary tumours $[0 \%$ NPV]). Therefore, cases with imaging performed at the authors' (tertiary) centre did not appear to be associated with markedly different performance than imaging from elsewhere (example shown in Figure 2). The study lacked power to answer this question, however. Performing a dedicated MRCP (rather than MR imaging alone) did not appear to improve the NPV: of 22 patients who had MR imaging with MRCP and had no mass apparent, 20 (91\%) ultimately were diagnosed with malignancy, yielding an NPV of only $9 \%$ for MRCP. 


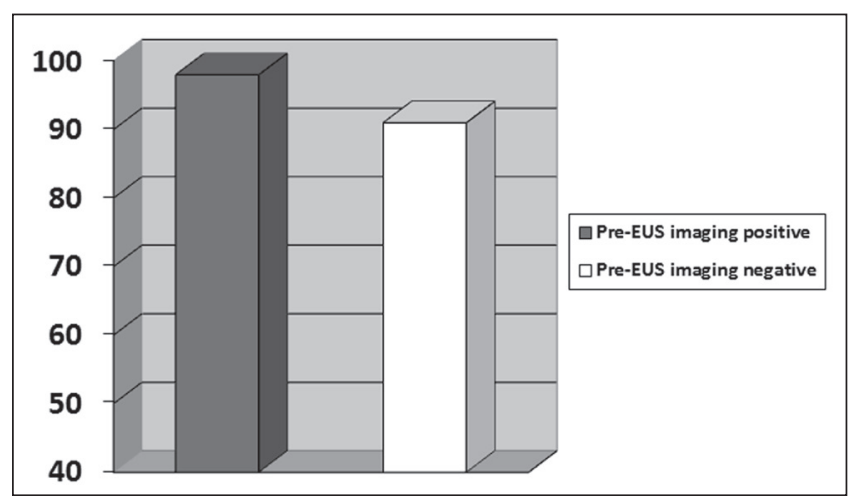

Figure 1) Percentage of malignant final diagnoses stratified according to pre-endoscopic ultrasound (EUS) imaging results finding a mass

Accuracy and diagnostic yield of EUS and EUS-FNA

The performance characteristics of EUS/FNA are summarized in Table 2, and stratified according to pre-EUS imaging in Table 3. EUS demonstrated a mass in $96 \%$ of cases in the imaging-positive group versus $85 \%$ of cases in the imaging-negative group $(P=0.09)$; that is, very little difference in yield if the imaging before EUS showed, or did not show, a mass. The sensitivity and accuracy of EUS, respectively, was $95 \%$ and $93 \%$ overall: $98 \%$ and $98 \%$ in the imaging-positive group, and $91 \%$ and $89 \%$ in the imaging-negative group. The overall PPV of EUS was $98 \%$, with an NPV of $50 \%$ (five of 10) (moderately helpful, considering the high $>90 \%$ prevalence clinical setting). Although slice thickness and resolution have been proposed as reasons for CT/MR imaging missing lesions, mean short-axis mass size was only slightly different between the two groups $(\mathrm{P}=0.07)$ : imagingpositive $24.7 \mathrm{~mm}$ versus imaging-negative $20.8 \mathrm{~mm}$. In addition, the stage of the tumours found was comparable between the two groups.

The yield of EUS-FNA (malignant or suspicious cytology) was $92 \%$ in the imaging-positive group, versus $62 \%$ (75\% of those with FNA) if pre-EUS imaging was negative $(\mathrm{P}<0.001)$ (Table 1). For EUSFNA, the overall sensitivity and accuracy was $90 \%$ and $96 \%$ in the imaging-positive group, and $83 \%$ and $84 \%$, respectively, in the imaging-negative group (Table 3). Of the 10 patients with false negative EUS-FNA, four had biliary stents in place and one had a percutaneous transhepatic cholangiography drain (50\% had a biliary tube) versus $62 \%$ of the overall cohort having biliary drains/stents. However, five $(50 \%)$ of the 10 had findings of chronic pancreatitis at EUS, compared with only $13 \%$ (14 of 104) overall in the study. FNA was performed slightly more often in the imaging-positive group: 49 of $51(96 \%)$ versus 44 of $53(83 \%)(P=0.052)$. There was no difference in the mean number of passes performed $(\mathrm{P}=0.78)$.

Nonmalignant causes for obstruction, such as common bile duct stones, were not apparent, likely due to mostly having painless presentations often with weight loss. In addition, a majority of the patients underwent MRCP, ERCP or both in the community before referral, which presumably would have detected stones.

\section{DISCUSSION}

Not surprisingly, EUS and EUS with FNA were highly sensitive and accurate, and had a high PPV and diagnostic yield in this cohort. However, perhaps surprising to some, this high yield was present regardless of the findings of pre-EUS cross-sectional imaging. Lack of a definite mass on pre-EUS imaging, despite advances in CT and MR imaging, has a very low NPV $(9.4 \%)$. The overall sensitivity $(51 \%)$ and accuracy $(53 \%)$ were also low. Lack of a clear mass (likelihood near 1.0) is clearly not sufficiently reassuring in this clinical setting. CT/MR imaging certainly still has a role in detecting ductal dilation in this setting (present in $72 \%$ of imaging-negative patients), and identifying liver metastases and gross vascular involvement.
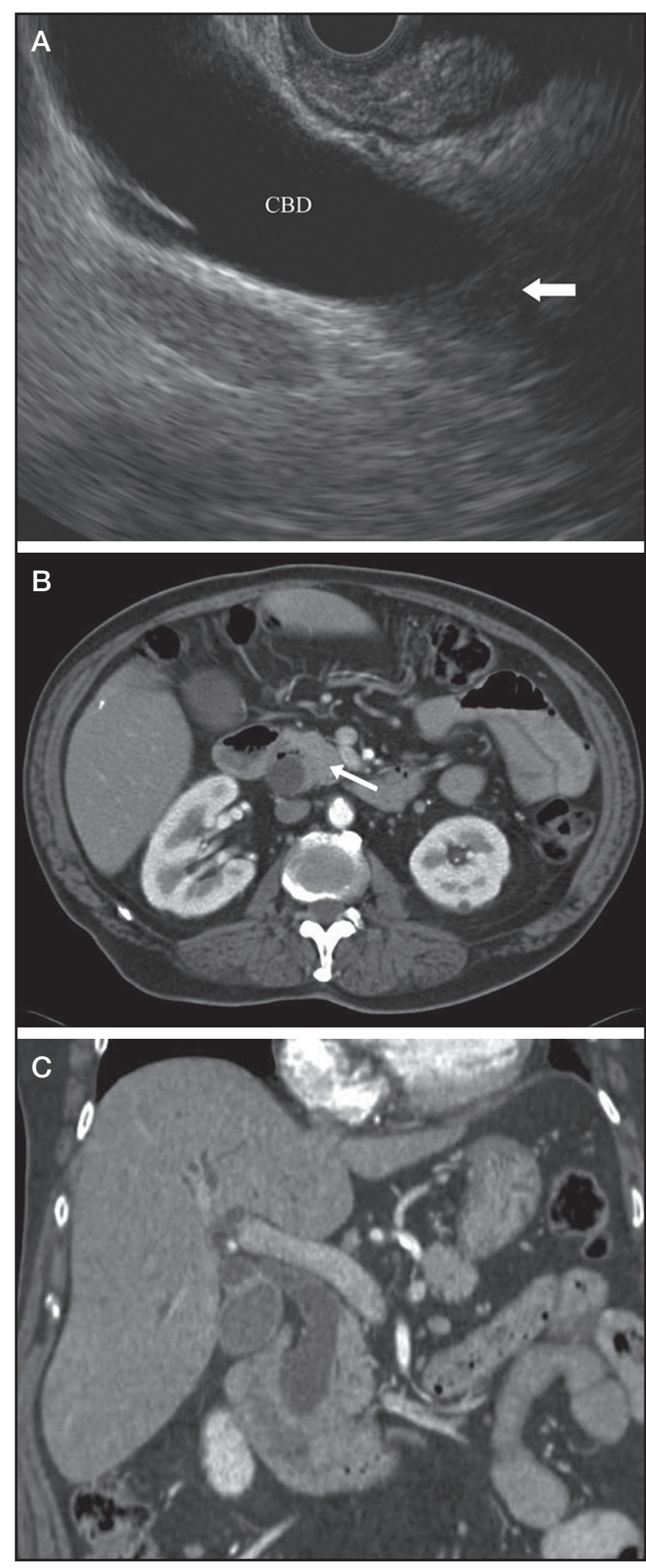

Figure 2) T3N1 periampullary pancreatic mass invading distal common bile duct (CBD) not apparent on high-quality pancreatic-protocol computed tomography (CT) performed at the authors' institution. Fine-needle aspiration showed cells suspicious for malignancy, confirmed at Whipple. On axial $\mathrm{CT}$, arrow shows isointense/isoperfused area in the head, into which the pancreatic duct ends, that in retrospect may represent the missed mass. Air near $\mathrm{CBD}$ is in a periampullary diverticulum. Endoscopic ultrasound in panel A. CT in panels B (axial) and C (coronal) 
Although efforts have been made to improve CT/MR resolution and slice thickness in the past decade, size of the lesion does not appear to be the issue - missed masses were only slightly smaller than the ones not missed, even when radiologists were given a suspicious clinical indication. It appears that the issue is more that the density/ signal/perfusion of pancreatic tumours, in many cases, is simply not significantly different from that of surrounding pancreatic parenchyma. It is unlikely, therefore, that further refinements in resolution or number of slices will resolve this problem.

Ultrasound, on the other hand, is an extremely accurate modality for detecting pancreatic masses, especially when chronic pancreatitis is not present. EUS takes that same excellent modality and eliminates factors that plagued conventional abdominal ultrasound (distance from probe to tumour, intervening fat and air), emerging in the past two decades as invaluable for detecting pancreatic cancer (2,6,9-12); EUS-FNA is a highly safe and accurate adjunct, obtaining tissue confirmation (2,13-17) and clarifying equivocal masses. Although older (before 2000) studies had shown comparability between CT and EUS $(5,19)$, this was confounded by tumours up to $7 \mathrm{~cm}$ in size being included in those studies. Sensitivity of CT in the small patient subgroup with smaller $(<2 \mathrm{~cm})$ tumours was modest (5). Despite this variable accuracy $(2,4-6)$, few small studies have ever evaluated EUS yield, after previous imaging was negative, in patients with a high clinical suspicion for pancreatic cancer $(2,15,20,21)$; low-suspicion cohorts had few cancers and, as such, lacked the power to show the higher miss rate of CT/MR imaging compared with EUS.

EUS test performance studies have presented limited to no data on test performance of previous imaging. In one study with low-suspicion patients, EUS found masses in $10 \%$ of CT-negative cases (16). In another, $72 \%$ of $25 \mathrm{CT}$-negative patients had cancer according to EUS (2). However, these may not be relevant, given the older (2000 to 2001) CT technology and small numbers, especially in the obstructive jaundice subgroup (2). A more recent (2006 [21]) albeit small caseseries found $90 \%$ of 10 CT-negative obstructive jaundice patients had masses on EUS, in keeping with our findings. The latter two studies did not address the value of MR. Our study included both MR and CT, in a more recent era and with a significantly larger sample size.

Even a very sensitive test in a very high prevalence setting will be anticipated to have only a modest NPV, as EUS-FNA did here $(2,13,17)$. Although EUS lowered the suspicion from $>90 \%$ (pre-test) to $50 \%$ (post-test), when negative, this is admittedly still not sufficiently reassuring in many patients, reminding providers to use caution even after negative EUS in high-suspicion patients. Exploring the reasons for EUS false-negatives, four of five had a final diagnosis of cholangiocarcinoma. In our analysis, we considered common bile duct 'thickening' to be negative, but it is sometimes the only EUS finding in this disease. The FNA yield in cholangiocarcinoma, unless there is a sizable mass (eg, at the hilum) (22,23), is known to be considerably lower than in pancreatic cancer $(24,25)$. Biliary drains/stents did not appear to affect FNA yield. However, patients with falsely negative EUS-FNA cytology were four times as likely to have chronic pancreatitis than the cohort overall.

One limitation of the present study was the lack of detail regarding the quality and protocols of the pre-EUS imaging, despite our efforts. The majority of the imaging studies were performed in the community and read by various radiologists before referral for EUS; however, this represents the real-life setting. The indication on these examinations was, however, obstructive jaundice and/or a suspicious biliary stricture, which should have led to a pancreatic protocol study; however, in many cases, we cannot be sure. The outside hospital films are routinely rereviewed by the EUS team, but not necessarily by a second radiologist. It is possible that, if reviewed again by a tertiary pancreatobiliary radiologist, a mass may have been able to be discerned despite outside radiologist and EUS endoscopist having missed it. That point may deserve further study; anecdotally, we have found that situation to be rare.

As noted above, the present study was a retrospective analysis without randomization or blinding, which are limitations. Although not a randomized comparison, the size and stage of the tumours found were comparable between the two groups. If blinding had been a significant confounder, one would have anticipated EUS to have performed significantly better when pre-EUS imaging was positive, leading the operator to make a positive diagnosis, but it did not. Another limitation of our study was the lack of surgical histology in some patients; however, we made every attempt to confirm the diagnosis using follow-up data in patients with negative EUS-FNA.

\section{CONCLUSION}

In the setting of suspicious biliary obstruction, EUS with FNA has a high yield regardless of the findings of 'real-life' pre-EUS crosssectional imaging. With the high pretest probability in this setting, EUS can likely be considered as a first-line test when available, perhaps saving the pancreatic-protocol CT to be ordered as needed by the treating cancer centre, avoiding the duplicate CTs that often occur in real life. A prospective and, perhaps, randomized trial of these imaging pathways may help to further clarify this issue. Regardless, lack of a mass on cross-sectional imaging, despite advances in the resolution of CT and MR imaging, has a very low NPV and an unhelpful likelihood ratio for a negative test (near 1.0), and is not sufficiently reassuring in this clinical setting.

SPONSOR/ACKNOWLEDGEMENT: JR was funded by the ASGE Senior Investigator Mentoring Award.

DISCLOSURES: JR (Olympus, consulting; Cook Medical, speaker/ honoraria).

\section{REFERENCES}

1. Hidalgo M. Pancreatic cancer. N Engl J Med;362:1605-17.

2. Agarwal B, Abu-Hamda E, Molke KL, Correa AM, Ho L. Endoscopic ultrasound-guided fine needle aspiration and multidetector spiral CT in the diagnosis of pancreatic cancer. Am J Gastroenterol 2004;99:844-50.

3. van der Gaag NA, Rauws EA, van Eijck CH, et al. Preoperative biliary drainage for cancer of the head of the pancreas. N Engl J Med 2010;362:129-37.

4. Muller MF, Meyenberger C, Bertschinger P, Schaer R, Marincek B. Pancreatic tumors: Evaluation with endoscopic US, CT, and MR imaging. Radiology 1994;190:745-51.

5. Legmann P, Vignaux O, Dousset B, et al. Pancreatic tumors: Comparison of dual-phase helical CT and endoscopic sonography. AJR Am J Roentgenol 1998;170:1315-22.

6. Mertz HR, Sechopoulos P, Delbeke D, Leach SD. EUS, PET, and CT scanning for evaluation of pancreatic adenocarcinoma. Gastrointest Endosc 2000;52:367-71.

7. Krishna NB, LaBundy JL, Saripalli S, Safdar R, Agarwal B. Diagnostic value of EUS-FNA in patients suspected of having pancreatic cancer with a focal lesion on CT scan/MRI but without obstructive jaundice. Pancreas 2009;38:625-30.

8. Fargnoli R, Fusi I. [Computerized tomography of pancreatic tumors]. Tumori 1999;85(1 Suppl 1):S3-5.

9. Helmstaedter L, Riemann JF. Pancreatic cancer - EUS and early diagnosis. Langenbecks Arch Surg 2008;393:923-7.

10. Hunt GC, Faigel DO. Assessment of EUS for diagnosing, staging, and determining resectability of pancreatic cancer: A review. Gastrointest Endosc 2002;55:232-7.

11. Akahoshi K, Chijiiwa Y, Nakano I, et al. Diagnosis and staging of pancreatic cancer by endoscopic ultrasound. Br J Radiol 1998;71:492-6.

12. Wiersema MJ. Accuracy of endoscopic ultrasound in diagnosing and staging pancreatic carcinoma. Pancreatology 2001;1:625-32.

13. Eloubeidi MA, Chen VK, Eltoum IA, et al. Endoscopic ultrasoundguided fine needle aspiration biopsy of patients with suspected pancreatic cancer: Diagnostic accuracy and acute and 30-day complications. Am J Gastroenterol 2003;98:2663-8.

14. Ryozawa S, Kitoh H, Gondo T, et al. Usefulness of endoscopic ultrasound-guided fine-needle aspiration biopsy for the diagnosis of pancreatic cancer. J Gastroenterol 2005;40:907-11. 
15. Chaya C, Nealon WH, Bhutani MS. EUS or percutaneous CT/ US-guided FNA for suspected pancreatic cancer: When tissue is the issue. Gastrointest Endosc 2006;63:976-8.

16. Agarwal B, Krishna NB, Labundy JL, Safdar R, Akduman EI. EUS and/or EUS-guided FNA in patients with CT and/or magnetic resonance imaging findings of enlarged pancreatic head or dilated pancreatic duct with or without a dilated common bile duct. Gastrointest Endosc 2008;68:237-42.

17. Harewood GC, Wiersema MJ. Endosonography-guided fine needle aspiration biopsy in the evaluation of pancreatic masses. Am J Gastroenterol 2002;97:1386-91.

18. Eloubeidi MA, Varadarajulu S, Desai S, Wilcox CM. Value of repeat endoscopic ultrasound-guided fine needle aspiration for suspected pancreatic cancer. J Gastroenterol Hepatol 2008;23:567-70.

19. Midwinter MJ, Beveridge CJ, Wilsdon JB, Bennett MK, Baudouin CJ, Charnley RM. Correlation between spiral computed tomography, endoscopic ultrasonography and findings at operation in pancreatic and ampullary tumours. Br J Surg 1999;86:189-93.

20. Van Der Gaag NA, Nio NY, Hoogmoed J, et al. The value of endoscopic ultrasound in detecting periampullary tumors after a negative CT. Gastrointest Endosc 2009;69:AB250(1).
21. Craanen ME, van Waesberghe JH, van der Peet DL, Loffeld RJ, Cuesta MA, Mulder CJ. Endoscopic ultrasound in patients with obstructive jaundice and inconclusive ultrasound and computer tomography findings. Eur J Gastroenterol Hepatol 2006;18:1289-92.

22. DeWitt J, Misra VL, Leblanc JK, McHenry L, Sherman S. EUS-guided FNA of proximal biliary strictures after negative ERCP brush cytology results. Gastrointest Endosc 2006;64:325-33.

23. Fritscher-Ravens A, Broering DC, Knoefel WT, et al. EUS-guided fine-needle aspiration of suspected hilar cholangiocarcinoma in potentially operable patients with negative brush cytology. Am J Gastroenterol 2004;99:45-51.

24. Harewood GC. Endoscopic tissue diagnosis of cholangiocarcinoma. Curr Opin Gastroenterol 2008;24:627-30.

25. Lee JH, Salem R, Aslanian H, Chacho M, Topazian M. Endoscopic ultrasound and fine-needle aspiration of unexplained bile duct strictures. Am J Gastroenterol 2004;99:1069-73. 


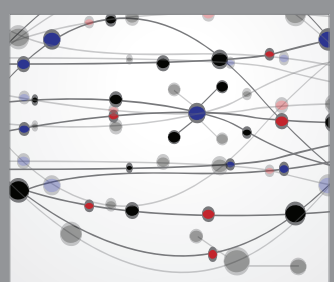

The Scientific World Journal
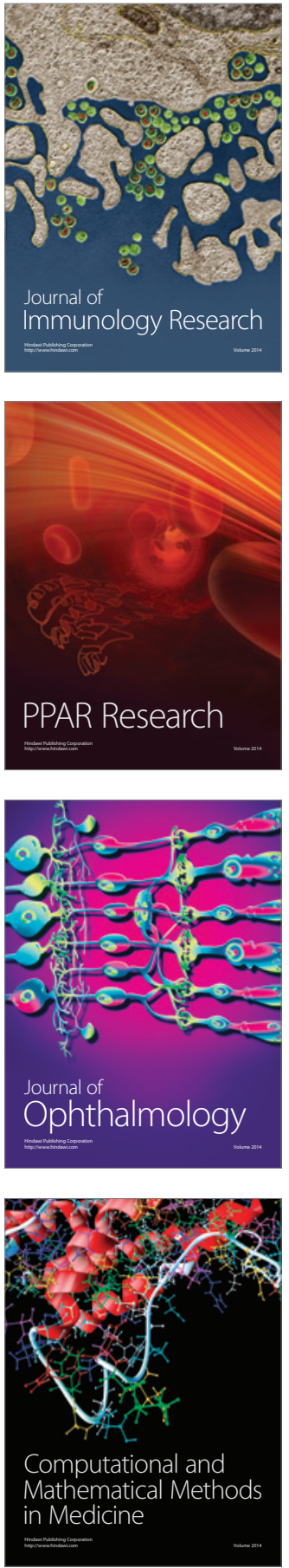

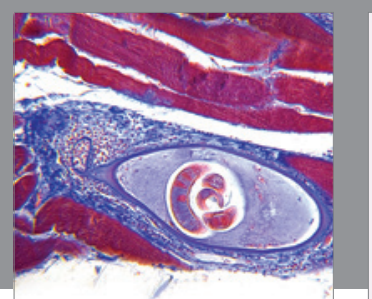

Gastroenterology Research and Practice

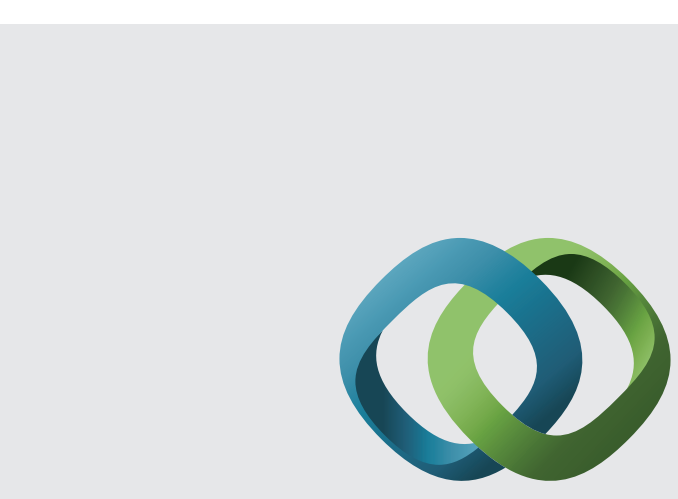

\section{Hindawi}

Submit your manuscripts at

http://www.hindawi.com
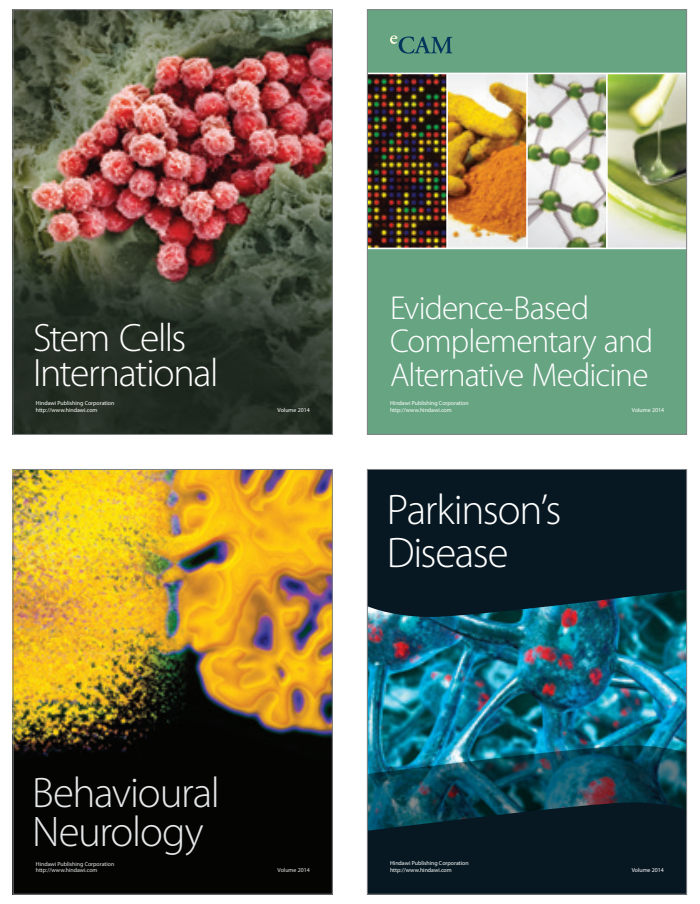
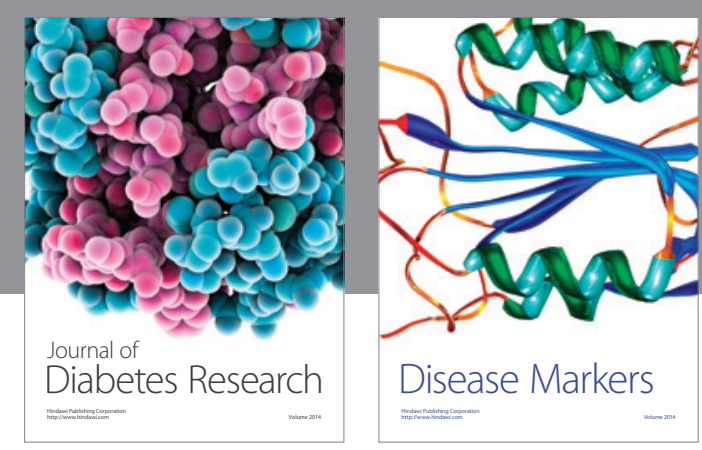

Disease Markers
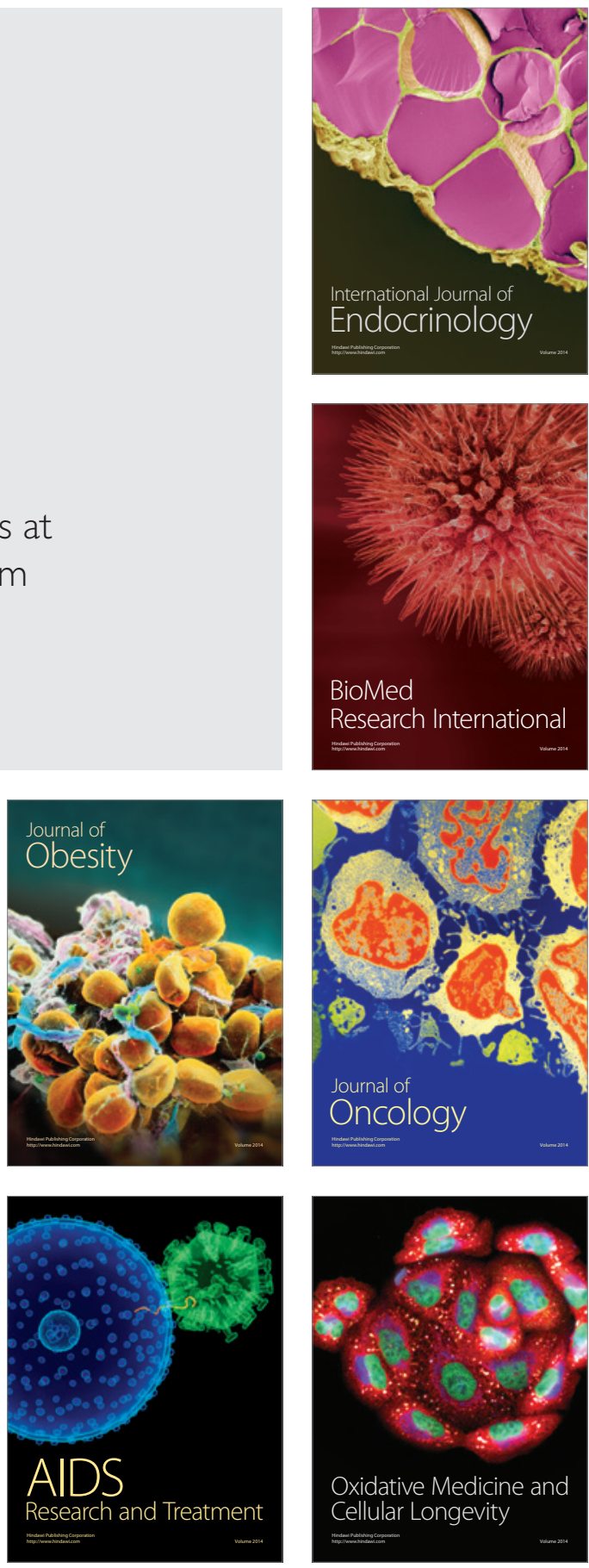